\title{
DETERMINACIÓN DE RIESGOS DE SALUD RELACIONADA A ESTILOS DE VIDA EN POBLACIÓN JOVEN
}

\section{DETERMINING HEALTH RISKS RELATED TO LIFESTYLES IN YOUNG PEOPLE}

Recibido 22 de septiembre 2015 Aceptado octubre 2015 Publicado Noviembre 2016

Correspondencia: Fernández Sánchez Abigail abifer2002@yahoo.com

\section{Autores:}

Abigail Fernández Sánchez

Profesor investigador de tiempo completo EESJ-UAEM

Ma. Guadalupe Ojeda Vargas

Rectora Campus Celaya Salvatierra UG

Ana Alicia García Carreño

ME EESJ-UAEM

Edith Ruth Arizmendi Jaime

Directora de la FE-UAEM

María Alejandra Terrazas Meraz

Profesor investigador de tiempo completo FE-UAEM

Palabras clave: Riesgos, Salud física emocional, Estilo de vida.

Keywords: Risks, emotional physical health, life style 


\section{RESUMEN}

Introducción: Diversos son los factores que generan differencias en el acceso al sistema de salud y su utilización, lo que produce desigualdades tanto en la promoción de la salud como en las posibililidades de la recuperación posterior una enfermedad.

Objetivo: El objetivo del estudio fue determinar los riesgos a la salud asociados a los estilos de vida y variables demográficas.

Metodología: Comesponde a un estudio descriptivo y correlacional, de orientación cuantitativa. La población de estudio estuvo constituida por 732 personas adolescentes y adulto joven, de ambos sexos, entre 17 y 33 años, estudiantes universitarios de nuevo ingreso. La muestra fue por conveniencia. Instrumentos: Escala Health-Promoting Lifestyle Profile II. General Health. SF-36. El procesamiento de la información se realizó con el Programa SPSS V-79. Resultados: Se encontró asociación significativa entre estilo de vida satisfacción, nutrición, relaciones interpersonales, actividad física y negativas con estrés.

Conclusiones: Los resultados permiten reafirmar la relevancia de considerar, los estilos de vida saludables como herramienta de valoración de salud física y mental para detectar posibles riesgos a la salud de los estudiantes de nuevo ingreso, en la mirada y en la intervención en salud para potenciar estilos de vida promotores de salud. Los estillos de vida se encuentran fuertemente asociados a riesgos que pueden ser evitables utilizando la promoción a la salud.

Palabras clave: Riesgos, Salud física emocional, Estilo de vida.

\section{ABSTRACT}

Introduction: Various factors that create differences in access to health care and utilization, resulting in inequalities in both health promotion and less chance the recovery an illness

Objective:Determine the risks associated with lifestyles and health and demographics variables.

Methodology: It is a descriptive and correlational study, quantitative orientation. The study population consisted of 132 adolescents and young adults, both sexes, between 17 and 33 years old, university freshmen. The sample was for convenience. Instruments: Scale Health-Promoting Lifestyle Profile II. General Health. SF-36. The information processing was performed using SPSS V-79. Results: significant association between satisfaction lifestyle, nutrition, relationships, physical activity and stress-negative was found.

Conclusions: The results allow us to reaffirm the importance of considering the healthy lifestyles as an assessment tool of physical and mental health to detect possible risks to the health of freshmen in the look and health intervention to boost life styles health promoters. Lifestyles are strongly associated with risks that may be avoidable using health promotion.

Keywords: Risks, emotional physical health, life style 


\section{INTRODUCCIÓN}

a decadencia de la salud en la poblaciónjoven es visible con estilo de vida demandante del siglo XXI. La etapa del ciclo vital tiene que ver en gran medida, en el cual se encuentre la persona así como la edad, género ya que determina el comportamiento de la persona, la cultura y el nivel socioeconómico conlleva a adoptar y/o llevar un estilo de vida en específico, en él favorecen las enfermedades crónicas desde temprana edad por el sedentarismo, nutrición, adicciones e inicio en la vida sexual activa, en una combinación de procesos emocionales y físicos

La atención primaria a la salud determina mejorar la problemática antes planteada, con intervenciones cuya esencia integradora en el individuo, familia o comunidad dirigida a corto mediano o largo plazo. En la medicina biologicista se planteó promover un estado óptimo de salud era un objetivo que debía colocarse en las acciones preventivas. Esto se constituyó como una novedad, pues identificó los factores que habían influido en la toma de decisiones y las acciones tomadas para prevenir la enfermedad como tal, pero la persona está inmersa en un entorno diverso que puede o no favorecer la salud?

La Promoción de la Salud se define como el proceso para proporcionar a los pueblos los medios necesarios para mejorar su salud y ejercer un mayor control sobre la misma (Carta de OHawa, 1986)

Por lo tanto el modelo de promoción de la salud en enfermería propuesto por Pender, según este modelo señala los determinantes de la promoción de la salud y los estilos de vida, están divididos en factores cognitivos-perceptuales, entendidos como aquellas concepciones, creencias, ideas que tienen las personas sobre la salud que la llevan o inducen a conductas o comportamientos determinados, el caso que nos ocupa, se relacionan con la toma de decisiones o conductas favorecedoras de la salud. La modificación de estos factores, y la motivación para realizar dicha conducta, lleva a las personas a un estado positivo llamado salud?
Por lo tanto el modelo de promoción a la salud desde 1996, de Nola Pender explica las características y experiencias individuales así como los conocimientos y afectos específicos de la conducta que llevan al individuo a participar o no en comportamientos de salud, cada uno en particular se encuentra inserto en un vínculo familiar y comunitario ${ }^{3}$.

Además, se identifica que los factores cognitivos perceptuales de los individuos, son modificados por las condiciones situacionales, personales e interpersonales, lo que da como resultado la participación en conductas favorecedoras de salud cuando existe una pauta para la acción.

El modelo de Pender es positivo, comprensivo y humanístico, toma a la persona como ser integral, analiza los estilos de vida, las fortalezas, la resiliencia son las potencialidades y las capacidades de la gente en la toma de decisiones con respecto a su salud y su vida.

Modelo Nola Pender

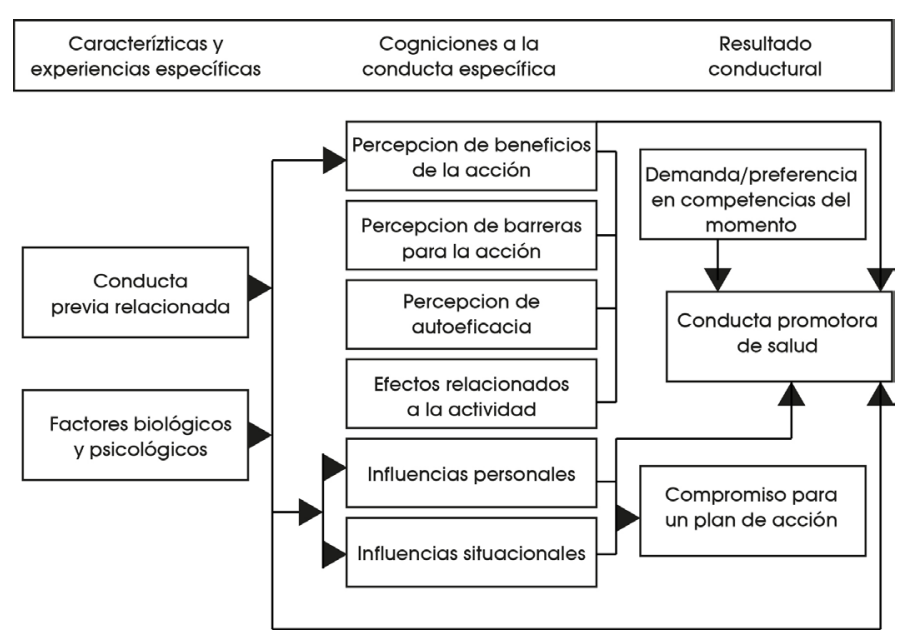

Modelo de promoción a la salud de 1996, de Nola Pender ${ }^{2}$

28

MMN Why Enf Neurol Vol. 15. No. 1 enero - abril 2016 
Este modelo le da importancia a la cultura, entendida ésta como el conjunto de conocimientos y experiencias que se adquieren a lo largo del tiempo, la cual es aprendida y transmitida de una generación a otra.

El modelo de promoción de la salud de Pender se basa en tres teorías de cambio de la conducta, influenciadas por la cultura, así:

La primera teoría, es la de la Acción Razonada: originalmente basada en Ajzen y Fishben, explica que el mayor determinante de la conducta, es la intención o el propósito que tiene la conducta de un individuo. Se plantea que es más probable que el individuo ejecute una conducta si desea tener un resultado. (Modelo parte izquierda)

Parte central del modelo. La conducta de una persona se realizará con mayor probabilidad, si ella tiene seguridad y control sobre sus propias conductas.

La Teoría Social-Cognitiva, de Albert Bandura en la cual se plantea que la auto-eficacia es uno de los factores más influyentes en el funcionamiento humano, definida como "Ios juicios de las personas acerca de sus capacidades para alcanzar niveles determinados de rendimiento". Adicional a lo anterior, la auto-eficacia es definida como la confianza que un individuo tiene en su habilidad para tener éxito en determinada actividad.

Según Pender², el Modelo de Promoción de la Salud retoma las características y experiencias individuales, además de la valoración de las creencias en salud, en razón a que estas últimas son determinantes a la hora de decidir asumir un comportamiento saludable o de riesgo para la salud, debido a su alto nivel de interiorización y la manera de ver la realidad que lo rodea. Martin fishbein importancia en los procesos cognitivos en el cambio de conducta. Basado en Bandura en el que la conducta es racional y económica².
La valoración de las creencias en salud relacionadas con los conocimientos y experiencias previas, determinan las conductas adoptadas por la persona; según el Modelo de Promoción de la Salud propuesto por Pender, estas creencias están dadas por:

Los beneficios de la acción percibidos o los resultados esperados, proporcionan motivación para adquirir una conducta de promoción de la salud, en la cual se da prioridad a los beneficios conductuales, destacando esta conducta entre otras personas, para que ellas puedan imitarla ${ }^{3}$.

La presencia de barreras para la acción, las cuales pueden ser personales, interpersonal salud desempeña un papel fundamental al determinar el estado de salud de cada persona, lo cual permitirá identificar las dificultades que se presentan y diseñar los mecanismos que permitan cambiar o disminuir una conducta de riesgo con el fin de mejorar la calidad de vida, para establecer un estado óptimo de salud a nivel físico, mental y social.

La auto-eficacia; Bandura ha encontrado en numerosos estudios, que las personas que se perciben así mismas competentes en un dominio particular realizarán repetidas veces la conducta en las que ellos sobresalen; la auto-eficacia es un sistema que provee mecanismos de referencia que permiten percibir, regular y evaluar la conducta, dotando a los individuos de una capacidad autorreguladora sobre sus propios pensamientos, sentimientos y acciones $^{3}$

Estados emocionales. Adaptación, motivación, relaciones sociales interpersonales. Las emociones, motivaciones, deseos o propósitos contemplados en cada persona promueven hacia una determinada acción. Los sentimientos positivos o negativos acompañados de un componente emocional son clave para identificar la conducta que necesita modificarse. Por lo tanto, en cada programa de salud deben implementarse actividades dinámicas y atractivas que generen beneficios para toda la población. 
Las influencias interpersonales y situacionales, son fuentes importantes de motivación para las conductas de salud, el impacto de las redes familiares y sociales o del entorno dentro del cual se desenvuelve la persona, pueden actuar positivamente generando un sentimiento de apoyo y aceptación, lo que brinda confianza a sus habilidades, esta sería una fuente valiosa para la creación de una conducta que promueva la salud; sin embargo, en el caso contrario, cuando el entorno familiar o social es adverso y nocivo, crea dificultades para adoptar dicha conducta, de ahí que sea a veces más conveniente cambiar algunas condiciones del medio social y económico, que apuntar al cambio de conducta en una persona.

Edad: particularmente tiene que ver en gran medida por la etapa específica del ciclo vital en la cual se encuentre la persona; a partir de la etapa en la que la persona se encuentre se verá afectado el estilo de vida.

Género: éste es un determinante del comportamiento de la persona, ya que el ser hombre o ser mujer hará que el individuo adopte determinada postura respecto a cómo actuar, además de lo que implica la prevalencia de algunas enfermedades que se verán reflejadas en mayor proporción en un género en específico.

Cultura: es una de las más importantes condiciones que llevan a las personas a adoptar un estilo de vida ya sea saludable o no; en ésta se incluyen los hábitos de alimentación, el tiempo de ocio y descanso, el deporte, entre otros, el descubrir las fortalezas de la cultura es de suma importancia en las formas de vivir desde nacer, crecer, trabajar y morir es el referente como obtenido su salud ${ }^{13}$.

Nivel socioeconómico: los recursos son un factor fundamental al momento de llevar un estilo de vida saludable, ya que si se pertenece a una clase media o alta se tendrán muchas más alternativas al momento de poder elegir una alimentación adecuada, y no sólo la alimentación sino también el acceso a la salud; mientras que para una persona de un nivel socioeconómico bajo, sus opciones se verán limitadas por la escasez de sus recursos económicos ${ }^{2}$.

Autoestima. Es la idea que se tiene del valor personal y el respeto que siente por sí mismo. La buena autoestima, solicitar ser tratado con respeto, atiende sus necesidades y defiende sus derechos. La baja autoestima, la persona se humilla y se coloca las necesidades de los demás antes de las suyas, piensa que no tiene nada que ofrecer. La otra parte importante de la autoestima es el de autoaceptación. Esto significa que reconoce y admite que todas sus partes, las deseables y las indeseables, las positivas y las negativas los puntos fuertes y los débiles se aceptan todo eso como un bloque que te hace un ser humano normal y valioso ${ }^{4}$

Autoeficacia académica, se define como la confianza que tiene el estudiante en su capacidad para dominar estrategias cognoscitivas de aprendizaje, manejar su tiempo y regular sus esfuerzos en esta área. Diversos estudios han mostrado cómo los estudiantes con altas expectativas de autoeficacia gozan de mayor motivación académica. Del mismo modo, obtienen mejores resultados, son más capaces de autorregular eficazmente su aprendizaje y muestran mayor motivación intńnseca cuando aprenden. En consecuencia, la mejora en las expectativas de autoeficacia, incrementa la motivación y el rendimiento en las tareas de aprendizaje ${ }^{5}$

Grado de urbanización. Los efectos de la urbanización y de la industrialización en los estilos de vida de las poblaciones rurales. Las presiones económicas y los incentivos que promueven la migración rural para áreas periféricas urbanas (más centros de salud, escuelas, canales de comunicación y más tiempo libre para actividades recreativas) determinan un mayor riesgo de obesidad. Por lo general, poblaciones que 
antes de migrar tenían actividad física con alto desgaste, asociada con la producción agńcola, se toman poblaciones urbanas marginales con menos trabajo físico; dieta desequilibrada, rica en grasa, energía y bebidas adulzadas; mayor consumo de alcohol y estilo de vida sedentario. Se explica que los seres humanos poseen tendencia natural a mantener el equilibrio energético, las alteraciones que promueven hábitos dietéticos inadecuados y que no estimulan la realización de actividad física aumentan el riesgo de afectar sus estilos de vida cotidiana y de otras enfermedades psicosociales ${ }^{6}$

Por último la tercer columna del modelo el resultado conductual, el individuo cuenta con libertad de elección, pero proveer las opciones es necesario por el personal de salud para lograr la aplicación del Modelo de Promoción de la Salud de Pender, es un marco integrador que identifica la valoración de conductas en las personas, de su estilo de vida, del examen físico y de la historia clínica, estas actividades deben ayudar a que las intervenciones en promoción de la salud sean pertinentes y efectivas y que la información obtenida refleje las costumbres, los hábitos culturales de las personas en el cuidado de su salud.

Los valores, actitudes, y conductas de salud se aprenden dentro del contexto familiar. Igual como los individuos deberán tomar responsabilidad por su propio estado de salud, así también las familias deberán tomar similares responsabilidades para la salud de la familia como unidad. Características estructurales y funcionales de la familia que se deberán tomar en cuenta al intentar influir en las prácticas de salud incluyen la estructura de valor, estructura de papel, estructura de poder (patrones en la toma de decisiones), patrones de comunicación, función afectiva, función de socialización, función de los cuidados de la salud, y función de manejo de estrés?

Múltiples situaciones ofrecen la oportunidad para la provisión de servicios para la promoción de la salud. Enfermeros, particularmente enfermeros con entendimiento de temas y problemas de la salud comunitaria, son idealmente adaptados para proveer liderazgo en el diseño, desarrollo, implementación, y evaluación de programas para la promoción de la salud en escuelas, sitios de trabajo, centros de enfermería, y otras situaciones comunitarias?

Apoyo económico. Los problemas de salud de hoy en día pueden mejorar al ser enfrentados principalmente, también al unirse con sectores en las sociedades para impactar las condiciones sociales y ambientales que comprometan a la salud. Los medios de comunicación y colaboración y, por lo tanto, poder para lograr soluciones que grupos u organizaciones que solos no tienen por sí mismos. Particularmente las escuelas o facultades de enfermería al unirse con otras facultades profesionales de salud, grupos de proveedores de cuidados de salud, y comunidades en la construcción de sociedades de salud. Estos arreglos, si diseñados de manera sensible a la comunidad, pueden mejorar los servicios de prevención y promoción de la salud brindada a diversas poblaciones?

El Modelo de Promoción de la Salud propuesto por Nola Pender, es ampliamente utilizado por los profesionales de enfermería, ya que permite comprender comportamientos humanos relacionados con la salud, y a su vez, orienta hacia la generación de conductas saludables. Tomando el mensaje:

La salud no tiene precio, la educación cuesta poco y vale mucho de Domingo Martín, yo agregaría el cuidado es humano tiene errores pero también aciertos, Abigail Fernández. 


\section{METODOLOGÍA}

E studio cuantitativo, descriptivo y correlacional. La población que participo fue de 132 adolescentes y adultos jóvenes 17 a 33 años, durante el año 2014, cuya muestra fue intencionada, incluyendo a los estudiantes universitarios de nuevo ingreso del estado de Morelos, realizando valoración psicosocial y sus estilos de vida relacionado a su salud. El estudio se llevo a cabo por profesores investigadores de la unidad, los instrumentos fue scaled version of the General Health Questionnaire 8 . Escalas y medidas de resumen del cuestionario SF-36 mide dos dimensiones y ochos escalas salud física (ESF-4) salud mental $(4 \mathrm{ESM})^{9}$, ajustando el instrumento con los estilos de vida recomendados por la escala Health-tilo Promoting Lifestyle Profile $\|^{10}$. Del instrumento el estilo de vida se aplican las dimensiones: Relaciones interpersonales, nutrición, responsabilidad en salud, actividad física y estrés. Cada indicador se califica con una puntuación de 1 a 5, donde 1 es nunca y 5 es siempre.

Para las variables demográficas se construyó un instrumento ad hoc integra: edad, sexo, estado civil, situación economía, adicciones y medidas antropométricas.

Consideraciones éticas, carta de consentimiento informado, se solicitó la firma del mismo informado a cada persona que reunía los requisitos para investigación.

Ley general de salud, clasifica en riesgo bajo ya que no se realizan intervenciones de riesgo.

Análisis de los datos se utilizó estadística descriptiva, coeficientes de correlación Pearson IC $\leq .05$, alpha de Cronbach para validación de confiabilidad del instrumento utilizado en nuestra población, encontrado alpha aceptable en las subescalas: Estrés de $\mathbf{\alpha}$.80, Satisfacción $\boldsymbol{\alpha}$.81. Relaciones interpersonales $\boldsymbol{\alpha}$.89. Salud $\boldsymbol{\alpha}$.92. Con uso del paquete estadístico SPSS versión 19.

\section{RESULTADOS}

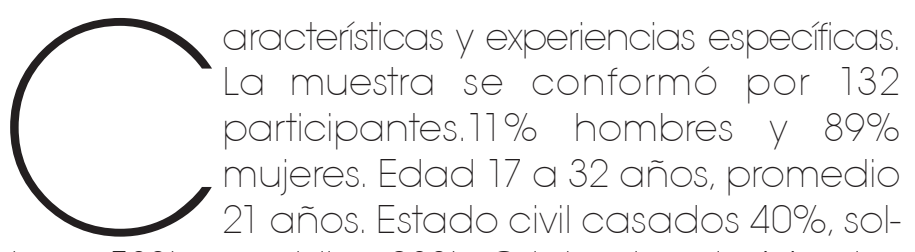
teros 53\%, con hijos 23\%. Originarios de Morelos 76\%, otros estados 24 Tipo de vivienda consideran cumple condiciones y servicios públicos 72\%, regulares condidiones 18\%, condidiones inadeavadas 10\%. Viven con su familia $91 \%$, viven con otras personas 9\%. Trabajan además de estudiar 12\%.

Consideran su dieta inadecuada y el comportamiento de su peso es: bajo 10\%, normal 63\% y sobrepeso obesidad 27\%.

Tabla 1. Economía y satisfacción, salud, estrés, relaciones interpersonales y nutrición del total de la población.

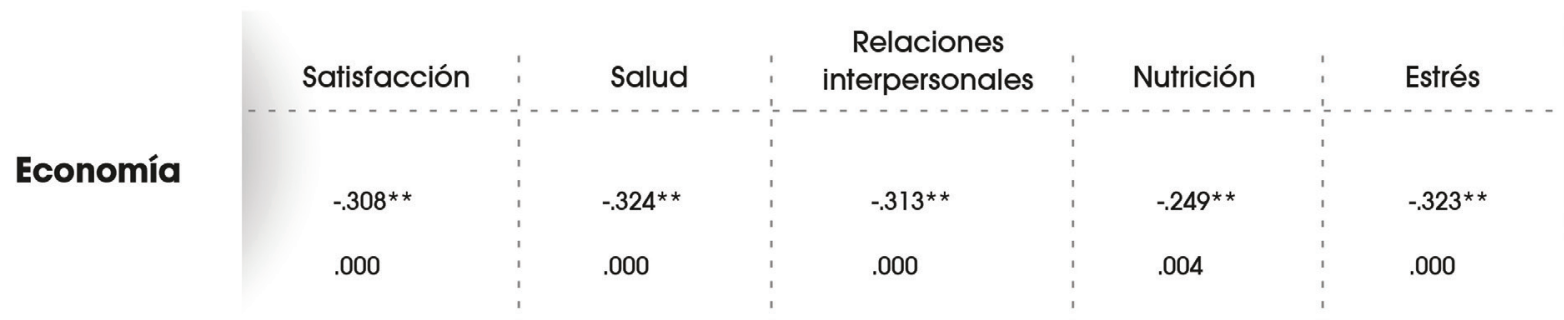

Se muestra en la tabla 1. La situación económica facilita, asegura y coloca a la persona en el alcance de los medios para cubrir sus necesidades básicas diarias satisfechas, por lo tanto menor condición económica afecta la satisfacción, salud, las relaciones interpersonales, nutrición e incrementa el estrés. 
Tabla 2. Nutrición y satisfacción, salud, seguridad y actividad física del total de la población.

\begin{tabular}{ll:l:c|c}
\multirow{3}{*}{ Nutrición } & Satisfacción & Salud & Seguridad & Actividad física \\
\hline & $.294^{\star \star}$ & $.350^{\star \star}$ & $.204^{\star \star}$ & $.179^{\star}$ \\
& .000 & .000 & .020 & .040
\end{tabular}

En la tabla 2, la prioridad de la nutrición de la persona significancias positivas necesidades satisfechas, para la salud como parte de seguridad y se relaciona con la actividad física.

Tabla 3. Relaciones interpersonales y satisfacción, salud y estrés del total de la población.

$\begin{array}{cc:c:c} & \text { Satisfacción } & \text { Salud } & \text { Estrés } \\ \text { Relaciones } & .527^{* *} & .454^{\star *} & -.392^{\star *} \\ \text { Interpersonales } & .000 & .000 & .000\end{array}$

En la tabla 3. Las relaciones interpersonales positivas y se cubren las necesidades tales como: satisfacción $r=.527^{* *}$ y salud $r=.454^{* *}$, sin embargo las significancias fueron negativas con presencia de estrés $r=-.392^{* *}$.

Tabla 4. Seguridad y estrés con: satisfacción, salud del total de la población.

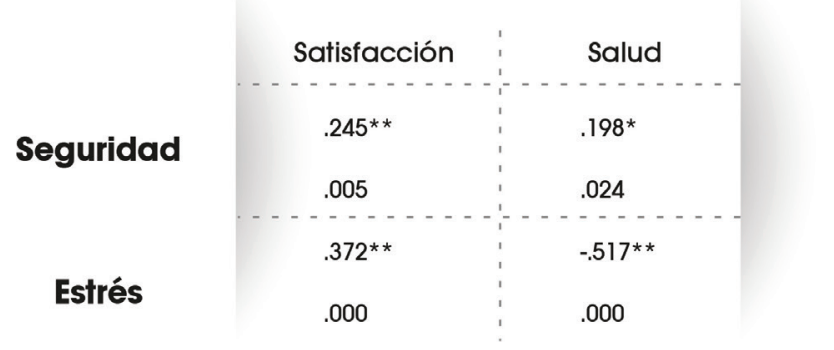

En la tabla 4. Las dimensiones seguridad relacionadas con satisfacción positivas y significativas $r=.245^{* *}$ y salud $r=.198^{*}$ son claras en sus puntuaciones positivas. Sin embargo el estrés afecta de modo negativo a las mismas variables satisfacción $r=-372 * *$ y salud $r=-.517 * *$

Tabla 5. Actividad física y salud con: satisfacción y adicciones del total de la población.

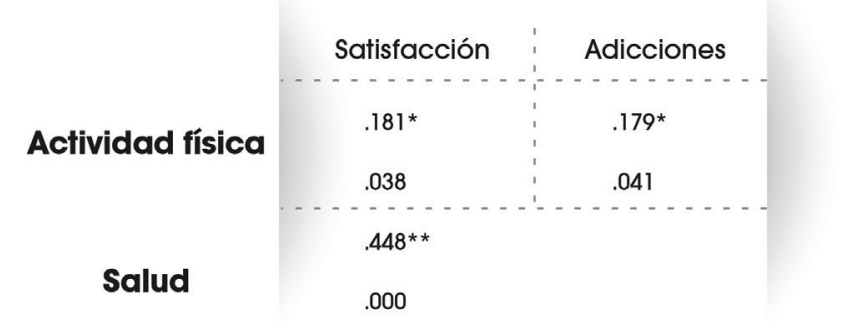

La tabla 5 indica la actividad física tiene una relación positiva con satisfacción $r=.187^{*}$ sin embargo se comporta similar las adicciones .179* se visualizan como una forma de relación con su medio ambiente propicio en las actividades físicas y adicciones. La salud y la satisfacción de manera simultánea muestra significancias positivas $r=.448^{* *}$ 


\section{DISCUSIÓN}

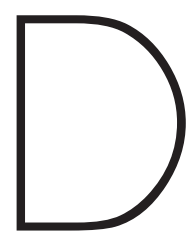

e acuerdo a Aristizábal y cols s se postula la importancia de los procesos cognitivos en el cambio de conducta e incorpora aspectos del aprendizaje cognitivo y conductual, reconoce que los factores psicológicos influyen en los comportamientos de las personas.

El factor económico, explica el valor de tener recursos que mantienen a la persona, la pobreza es determinante esencial para cubrir las necesidades y mantenerse al margen pues los participantes no cuentan con recursos relacionados al desempeño de su vida cotidiana.

La práctica y/o hábitos alimenticios guardan relación positiva la salud, seguridad actividad física en su vida cotidiana. La alimentación es significativa en relación a las actividades y desempeño de su vida cotidiana así como mantener su salud.

Según estudio de Rodríguez y colaboradores ${ }^{11}$ en su estudio calidad de vida y ejercicio físico muestra puntajes aceptables, de la misma manera las condiciones los resultados en este estudio muestran la interrelación con el cuidado de la salud de manera muy positiva, así mismo la satisfacción con las actividades de la vida cotidiana que realizan guardan relación positiva la salud emocional y física, actividad física, nutrición y el estado de ánimo.

En relación a la actividad física los informantes reconocen su importancia, pero no hay relaciones significativas con adicciones y con los recursos de ellos para llevar una vida activa con la práctica de ejercicio.

En relación a las adicciones no se relacionan con las variables solo con actividad física, (posiblemente por el tipo de convivencia) sin embargo gran numero de los entrevistados ingieren bebidas con alcohol y un número menor fuman, no mencionan consumir algún tipo de droga.
Relaciones interpersonales. En relación a las emociones positivas mantienen relaciones interpersonales como actitudes positivas en relación a su salud, pero también se encuentran involucradas las actitudes negativas que conllevan a la baja autoestima y minusvalía. De acuerdo con estudios relacionados a encontrar el sentido de la propia vida, percibir y experimentar que la vida está bajo control personal, que es libre y autodeterminativo ${ }^{12}$.

\section{CONCLUSIONES}

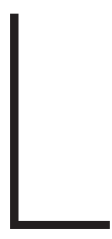

a teoría de Pender expresa que la conducta está motivada por el deseo de alcanzar el bienestary el potencial humano. La conducta es racional, considera que el componente motivacional clave para conseguir un logro. De acuerdo con esto, cuando hay una intención clara, concreta y definida por conseguir una meta.

El modelo de promoción a la salud expone las características y experiencias individuales así como los conocimientos y afectos específicos de la conducta que llevan al individuo a participar o no en comportamientos de salud ${ }^{3}$.

En las conductas para la promoción de salud participa regularmente la familia, y el equipo multidisciplinario para contar con un bienestar propio a las demandas físicas e intelectuales que el estudiante requiere.

Fomentar la promoción de la salud particularmente con los todos miembros de la familia participe para favorecer conductas o los patrones y estilos de vida.

Consistencia entre valores familiares de salud como detectar los riesgos y que acciones de salud son pertinente así como las metas explícitas o implícitas de la familia en el área de la salud.

Las evaluaciones diagnósticas que se realizan a los estudiantes guardan relación con niveles de aprendizaje previo, se ha descartando los medios

\section{4 4MN/Why Enf Neurol Vol.15. No. 1 enero - abril 2016}


y recursos por los que ellos aprenden y que deben tomar en cuenta y de algunas formas son los estilos de vida que contribuyen a riesgos en la salud física, emocional y mental necesarios en su cotidiano como estudiante y el profesionista del futuro. Por lo tanto nos guie a nuevas investigaciones que exploren la relación a la promoción de la salud como un modelo que asegure los estilos de vida saludables de la familia como estrategia, así como los valores, creencias, percepción del cuidado y la prevención de riesgos de la salud física y mental.

\section{REFERENCIAS BIBLIOGRÁFICAS}

1. Arredondo A. Análisis y relexión sobre los modelos teóricos del proceso salud-enfermedad. Cad. Saúde Pública (serie en línea). 1992; 254,261. (citado 2015). Disponible en:

2. Pender, N. J. Heath Promotion Model Manual 2011; 4-17

3. Aristizábal GP., Blanco DM, Sánchez A., Ostiguín RM. El modelo de promoción de la salud de Nola Pender. Una relexión en torno a su comprensión Rev. Enfermería Universitaria ENEO-UNAM 2011. 8 (4); 17-23.

4. Larraburu I. Atención plena ed. España. 2015

5. Gutiérrez A., Vega A., Jara E., Faúndez F., Valassina F. Evaluaciones diagnósticas aplicadas a estudiantes que ingresan a primer año de universidad. Ministerio de Educación. Gobierno de Chile 2009; p. 31

6. Peña M., Bacallao J. Obesidade e pobreza: um novo desafio à saúde pública. São paulo: roca, 2006.

7. Giraldo A., Toro MY., Macías AM., Valencia CA., Palacio S. La promoción de la salud como estrategia para el fomento de estilos de vida saludables. Rev SCielo. 2010.
8. Goldeberg, D.P. \& Hillier, V.F. (1979). A scaled version of the General Health Questionnaire. Psychol Med, 9, 139-45.

9. Olivares y Tirado. Escalas y medidas de resumen del cuestionario SF-36. 2006, Chile.

10. Walker, Susan N.; Sechrist, Karen R.; Pender, Nola J.Health Promotion Model - Instruments to Measure Health Promoting Lifestyle : HealthPromoting Lifestyle Prolle (HPLP II) (Adult Version)

11. Rodríguez MC., Molina J., Jiménez C. y Pinzón T. Calidad de vida y actividad física en estudiantes, docentes y administrativos de una universidad de Bogotá. Cuadernos hispanoamericanos de psicología, 2011.11 (1) 19-37.

12. García Gallego, JF., Pérez E. Sentido de la vida y desesperanza: un estudio empírico. Rev. Universitas psychologica 2005; 8 (2), 447 454.

13. Vidal D., Chamblas I., Zavala M., Müller R., Rodríguez MC., Chavez A. Determinantes sociales en salud y estilos de vida en población adulta de Concepción, Chile Rev. Ciencia y enfermería. 2014. XX (1): 61-74. 